\title{
ARTICLE \\ Diazepam reverses increased anxiety-like behavior, social behavior deficit, and dopamine dysregulation following withdrawal from acute amphetamine
}

\author{
Millie Rincón-Cortés $\mathbb{D}^{1,2}$, Kimberly G. Gagnon ${ }^{1}$, Hannah K. Dollish ${ }^{1}$ and Anthony A. Grace (iD ${ }^{1,2,3}$
}

\begin{abstract}
Psychostimulants such as amphetamine (AMPH) increase dopamine (DA) release from ventral tegmental area (VTA) neurons, which is associated with their acute reinforcing actions. This positive state is followed by a negative affective state during the withdrawal period each time the drug is taken (i.e., opponent process theory). AMPH withdrawal is accompanied by symptoms of anxiety and depression, which are associated with DA system dysfunction in humans and animal models. Most studies have focused on the negative affective state after withdrawal from chronic drug administration; yet, this negative state appears even after a drug is taken for the first time in both humans and rodents. In rats, withdrawal from a single dose of AMPH (2 mg/kg) increases forced swim test immobility and decreases the number of spontaneously active VTA DA neurons up to $48 \mathrm{~h}$ post-withdrawal. In the current study, acute AMPH withdrawal was found to increase anxiety-like behavior in the elevated plus maze (EPM), reduce social cage time in the three-chambered social approach test (SAT), and attenuate VTA population activity. The effects of diazepam, a drug commonly used to treat anxiety disorders, were tested on anxiety-like and social behavior as well as VTA DA neuron activity following acute AMPH withdrawal. A single $(5 \mathrm{mg} / \mathrm{kg})$ dose of diazepam circumvented the neurobehavioral effects induced by acute AMPH withdrawal, as demonstrated by increased open arm time and social cage time as well as normalized VTA DA activity comparable to controls, suggesting that these neurobehavioral effects of acute AMPH withdrawal reflect an anxiety-like state.
\end{abstract}

Neuropsychopharmacology (2018) 43:2418-2425; https://doi.org/10.1038/s41386-018-0123-8

\section{INTRODUCTION}

Withdrawal from drugs of abuse is associated with symptoms of negative affect, including loss of motivation for natural rewards or anhedonia, depression, and anxiety, as well as dysregulation of brain reward systems involving some of the same neurochemical systems implicated in the acute reinforcing drug effects [1]. Although psychostimulants such as amphetamine (AMPH) exert their acute reinforcing actions through mesolimbic system activation and increasing dopamine (DA) release from ventral tegmental area (VTA) neurons [2,3], this positive emotional state is followed by a negative affective state each time the drug is taken (i.e., opponent process theory) [4, 5]. Amphetamine withdrawal (AMPH-W) is accompanied by symptoms of anxiety and depression in humans and rodents, which are commonly associated with DA system dysfunction [6-10]. Although most studies have focused on the negative affective state after withdrawal from long-term (i.e., chronic) drug administration, the negative affective state appears even after a drug is taken for the first time $[11,12]$.

In rats, withdrawal from an acute single ( $2 \mathrm{mg} / \mathrm{kg}$ ) dose of AMPH increases immobility in the forced swim test (FST) and attenuates the number of spontaneously active DA neurons (i.e., population activity) in the VTA [13]. These findings are consistent with the effects of chronic mild stress (CMS), a rodent model of stressinduced neurobehavioral alterations relevant to depression, which results in higher levels of anxiety-like behavior and FST immobility as well as decreased VTA population activity $[14,15]$. CMS effects on immobility behavior and VTA DA activity are reversed by ketamine administration [15], which also elicits antidepressant-like effects on behavior and the DA system in the learned helplessness model of depression [16]. In the context of withdrawal from an acute single dose of AMPH, however, there was a dissociation: ketamine reversed the DA decrease but had no effect on FST immobility [13]. This finding suggests that VTA DA neuron activity and FST immobility are correlated but not interdependent and raises the possibility that different processes may be present between some depressive-like behaviors and acute AMPH-W.

Acute withdrawal from drugs of abuse, including psychostimulants, can produce an anxiety-like state [17-19]. In humans, anxiety is one of the most prominent symptoms of psychostimulant withdrawal during the first several weeks of abstinence [20]. In rodents, short-term (24-48 h) withdrawal from psychostimulants (i.e., cocaine, AMPH) increases anxiety-like behavior in the elevated plus maze (EPM) [10, 21]. Clinical and basic studies suggest a link between anxiety and social dysfunction [22, 23]. For example, impairments in social functioning have been reported in patients with generalized anxiety disorder [24, 25]; and benzodiazepines, including diazepam, can ameliorate social disturbances in clinical anxiety and are commonly used to treat anxiety disorders [26-28]. In rodents, anxiolytics prevent decreases in

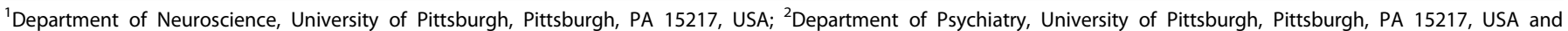
${ }^{3}$ Department of Psychology, University of Pittsburgh, Pittsburgh, PA 15217, USA

Correspondence: Millie Rincón-Cortés (mir69@pitt.edu)

Received: 13 January 2018 Revised: 8 June 2018 Accepted: 12 June 2018

Published online: 18 June 2018 
social interaction that occur in response to anxiogenic environments (i.e., novel environments, bright light) [22]. Although drug withdrawal is associated with alterations in the social domain [29, 30], the effects of withdrawal from an acute single dose of AMPH on anxiety-like and social behaviors in rodents remain poorly understood. Given that high anxiety levels are frequently associated with alterations in the social domain, we hypothesized that acute AMPH-W would increase anxiety-like behavior and impair social behavior. Consistent with our hypothesis of acute AMPH-W inducing an anxiety-like behavioral state, we predict it would respond to diazepam (i.e., antianxiety agent) treatment.

The VTA is activated by drugs of abuse and natural rewards and plays an important role in motivated social behavior [31-33]. Similar to drugs of abuse, social encounters are rewarding to rodents and social stimuli increase neural activity in the VTA [34]. Dysregulation of dopaminergic activity has been linked to impairments in social behavior [35] and increased anxiety-like behavior in rats [14]. Neuroimaging studies in humans have revealed decreases in DA during psychostimulant withdrawal, which are thought to contribute to the dysphoric state observed $[8,17]$. In line with this notion, previous work from our group has demonstrated that withdrawal from acute $\mathrm{AMPH}$ reduces the activity of VTA DA neurons $18-48 \mathrm{~h}$ postinjection [13]. Based on these data, we propose a link between increased anxiety-like behavior, reduced social behavior, and a hypodopaminergic state following acute AMPH-W, which is thought to underlie the negative affective state. Given our hypothesis that diazepam will be able to reduce anxiety-related behaviors induced by acute withdrawal from a single dose of $\mathrm{AMPH}$, we predict that these antianxiety effects will be reflected in VTA DA neuron activity and that diazepam administration will also normalize DA system function.

\section{MATERIALS AND METHODS}

Animals

Adult male Sprague Dawley rats (300-325 g at arrival; Envigo, Indianapolis, IN; total $n=118$ ) were housed in pairs in a reverse 12-h light/dark cycle and temperature-controlled room with food and water available ad libitum. All experiments were performed with accordance to the guidelines outlined in the National Institutes of Health Guide for Care and Use of Laboratory Animals and were approved by the Institutional Animal Care and Use Committee of the University of Pittsburgh.

Drug injections

Amphetamine. Acute AMPH-W was modeled by injecting animals with AMPH (2 mg/kg intraperitoneally (i.p.)), with controls receiving saline (SAL; $1 \mathrm{ml} / \mathrm{kg}$ i.p.), $18 \mathrm{~h}$ prior to behavioral testing. All recordings were conducted $18-40 \mathrm{~h}$ post-AMPH administration. The dose and time course were selected based on previous findings from our group indicating enhanced FST immobility and attenuated VTA population activity $18-48 \mathrm{~h}$ post-AMPH injection [13].

Diazepam. Animals received either SAL vehicle $(1 \mathrm{ml} / \mathrm{kg})$ or diazepam ( $5 \mathrm{mg} / \mathrm{kg}$ ) $30 \mathrm{~min}$ prior to behavioral testing. This dose was selected based on prior work by our group indicating its effectiveness at reducing anxiety-like behavior in adult male rats in a neurodevelopmental model of schizophrenia when administered acutely [36] and in a stress-induced social avoidance model of anxiety in adult male rats [37].

Elevated plus maze

The EPM was positioned $50 \mathrm{~cm}$ above the floor and consisted of a plus-shaped apparatus composed of 4 arms (2 enclosed arms opposed by 2 open arms; each $50 \mathrm{~cm}$ long $\times 10 \mathrm{~cm}$ wide) [38]. Each rat was placed on the central platform and its movement was recorded for $5 \mathrm{~min}$ with a camera positioned overhead. The time spent on open arms (s), defined as front two paws and head in the arm, and the number of open arm entries were measured as indices of anxiety-like behavior by an experimenter blind to treatment status; the total number of entries was used as an index of locomotor activity. EPM testing occurred $18 \mathrm{~h}$ following SAL or AMPH injection and animals were habituated to the testing room, along with other animals in the same cohort to be tested that day, for at least $1 \mathrm{~h}$ prior to testing, which occurred during the dark phase of the light-dark cycle. The arms and side walls of the apparatus were cleaned with $70 \%$ ethanol and dried between subjects.

\section{Social behavior test}

A three-chambered apparatus was used to assess social approach behavior, also known as social motivation $[39,40]$. In this test, a rat is placed in a smaller center chamber in between two larger chambers, each of which contains a receptacle that allows the animal to see and smell its content but not contact it directly, thereby preventing confounding factors of aggression and fighting. Test rats were acclimated to the testing room for at least $1 \mathrm{~h}$ prior to testing and given a 5-min habituation in which the rat was placed in the center chamber and allowed to explore the testing apparatus freely. After habituation, an unfamiliar younger samesex rat (i.e., social stimulus), which had been previously habituated to the wire cage inside the testing apparatus for 15 min under same testing conditions, was enclosed inside a wire cage and placed in a side chamber. An empty wire cage containing an inanimate object was placed in the opposite chamber as a novel object control. The experimental rat was then allowed to explore the entire apparatus and the time spent sniffing the receptacle containing the social stimulus (i.e., social cage) was recorded by a blind experimenter for $10 \mathrm{~min}$. Owing to our limitations to capture actual inhalation, the primary measure of sociability is used here is social sniff time and refers to the time the target (i.e., test) rat spent in close proximity to the social stimulus cage with its snout directed toward the cage (i.e., cage time), which is consistent with prior studies assessing sociability in rodents $[39,41]$. This measure is a more reliable measure of AMPH effects on rodent social behavior and has higher test-retest reliability and validity when compared with chamber scores [39, 41, 42]. Percentage change from baseline in social cage time was calculated for each animal using Excel [difference between the two social cage times (i.e., baseline, posttreatment) divided by the original (i.e., baseline) value, which is then multiplied by 100 to obtain a percentage].

The cohort of animals was exposed to a 5-min habituation period followed by a 10-min test to assess baseline sociability (Day 1) followed by a second 10 -min test (Day 2) $18 \mathrm{~h}$ post SAL or AMPH injection. Effects of diazepam $(5 \mathrm{mg} / \mathrm{kg})$ on social behavior during $\mathrm{AMPH}-\mathrm{W}$ were assessed in separate cohorts of animals that underwent the same procedure, except for an injection of vehicle or drug (i.e., diazepam) injection 30 min prior to testing on Day 2. All testing occurred during the dark phase of the light-dark cycle and the chambers of the apparatus were cleaned and dried between each subject. The same experimental and social stimulus animal were never paired more than once and behavioral scoring was conducted blind to treatment status.

\section{VTA DA neuron recordings}

Single-unit extracellular recordings were performed using an acute preparation as previously described [15] in the same animals that had undergone drug administration and the social behavior testing. Briefly, rats were anesthetized with $8 \%$ chloral hydrate (400 mg/kg, i.p., Sigma) and placed in a stereotaxic apparatus (Kopf). Body temperature was kept at $37^{\circ} \mathrm{C}$ and the level of anesthesia were constantly monitored; anesthesia was adjusted by administration of additional chloral hydrate as needed. A burr hole was drilled in the region overlying the VTA on the right side of the 
EPM
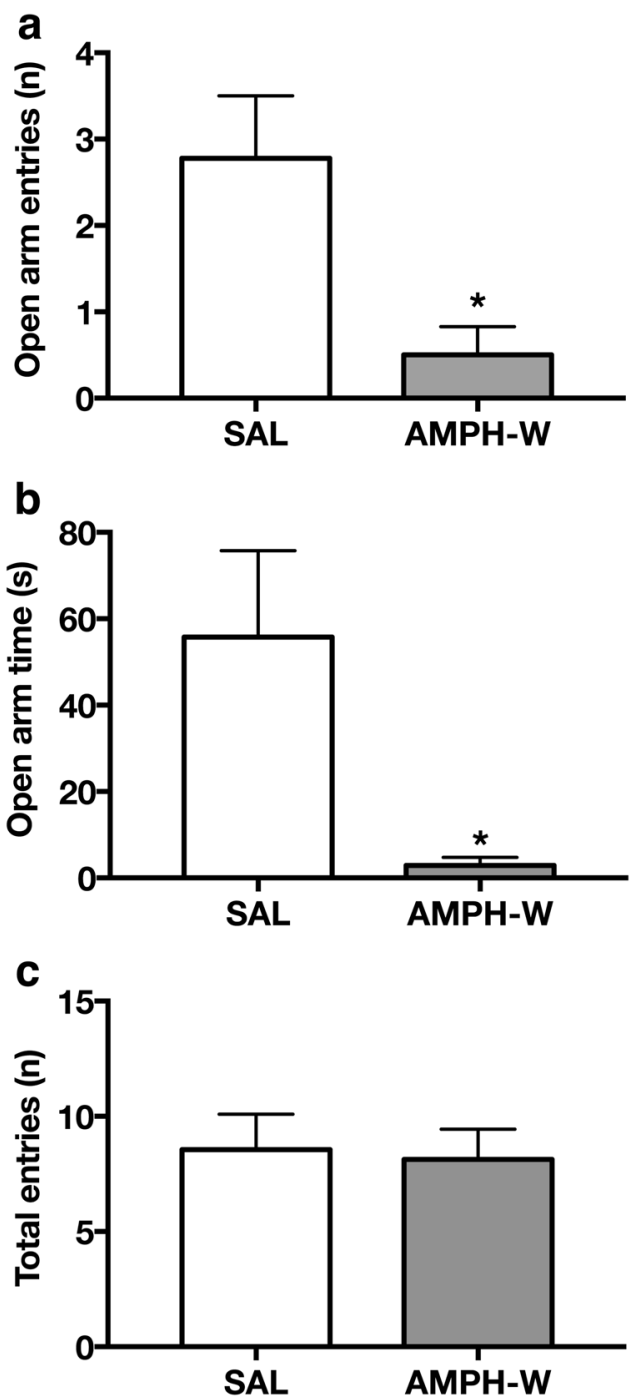

Fig. 1 Withdrawal from acute AMPH increases anxiety-like behavior. Compared with saline (SAL) controls $(n=9)$, rats that had undergone amphetamine withdrawal (AMPH-W) $(n=8)$ exhibited a lower number of open arm entries $(p<0.05)$ and $\mathbf{b}$ less time spent in the open arm $(p<0.05)$ but $\mathrm{c}$ no difference in the total number of entries $(p=0.84) .{ }^{*} p<0.05$. Error bars indicate mean + SEM

brain. Glass electrodes were lowered into the VTA using a hydraulic microdrive (Kopf) and population activity was determined by counting the number of spontaneously active DA neurons encountered while making 6-9 vertical electrode passes (i.e., tracks), each separated by $200 \mu \mathrm{m}$, in a predetermined grid pattern that spans the antero-posterior (AP) and medio-lateral (ML) extent of the VTA (AP: $5.3-5.7 \mathrm{~mm}, \mathrm{ML}: 0.6-1.0 \mathrm{~mm}$ from bregma, and dorsoventral (DV): $6.5-9.0 \mathrm{~mm}$ from dura). Spontaneously active DA neurons were identified with open filter settings using well-established electrophysiological criteria, including location, slow, irregular, firing pattern, and long duration; variable shape biphasic action potential waveform $(>2.2 \mathrm{~ms})$; and half width (>1.1 ms), among others [43, 44]. Once identified, DA neurons were recorded for $3 \mathrm{~min}$ (1-min minimum) and three parameters of DA activity were measured: (1) population activity (i.e., number of spontaneously active DA neurons per electrode track); (2) basal firing rate; and (3) the proportion of action potentials occurring in bursts [burst initiation defined as the occurrence of two spikes with an interspike interval of $<80 \mathrm{~ms}$, and the termination of the burst defined as the occurrence of an interspike interval of $>160 \mathrm{~ms}$ ] [45]. Electrode placement was verified via electrophoretic ejection of Chicago Sky Blue dye (Sigma) at the last recording site (see Supplemental Methods and Supplementary Figure S1).

Statistical analyses

For behavioral testing, pairwise comparisons were analyzed using unpaired, two-tailed $t$ tests; comparisons between three or more groups were analyzed using one-way analysis of variance (ANOVA). Percentage change from baseline in social cage time was analyzed using a two-way ANOVA. Electrophysiological data of DA neurons was collected with Powerlab Lab Chart (AD Instruments) to identify spike time courses and exported to the Neuroexplorer (NEX Technologies, NexTech Systems) software to calculate firing rate and burst firing. Electrophysiological data were analyzed using two-way ANOVA. Post hoc analyses (i.e., Tukey's multiple comparison post hoc test) were performed for ANOVAs indicating a main effect and two-way ANOVAs indicating an interaction. All statistics were calculated using GraphPad Prism 7.0 and differences were considered significant when $p<0.05$.

\section{RESULTS}

Acute withdrawal from a single dose of AMPH increases anxietylike behavior

To determine the effects of acute AMPH-W on anxiety-like behavior, a cohort of rats was tested $18 \mathrm{~h}$ postinjection (SAL or AMPH) in the EPM (SAL: $n=9$, AMPH-W: $n=8$ ). Amphetaminewithdrawn animals made fewer of entries into the open arms $(p<$ $\left.0.05 ; t_{(15)}=2.751\right)$ (Fig. 1a) and spent less time in the open arm ( $p$ $<0.05 ; t_{(15)}=2.476$ ) (Fig. 1b) compared with SAL controls. The total number of crossings did not differ between groups $(p=0.84$; $\left.t_{(15)}=0.21\right)$ (Fig. 1c).

Acute withdrawal from a single dose of AMPH reduces social motivation

Rats were randomly assigned to treatment groups and tested for social behavior prior to drug exposure to establish baseline levels of social behavior and ensure that there were no preinjection differences between groups (SAL: $n=9$, AMPH: $n=7$ ). To assess effects of acute AMPH-W on sociability, these same animals were tested again $18 \mathrm{~h}$ postinjection. Both groups were comparable at baseline (i.e., preinjection) (Fig. 2a) and showed similar levels of social cage sniff time $\left(p=0.50 ; t_{(14)}=0.68\right)$. Eighteen hours postinjection, AMPH-treated rats exhibited a reduction in social cage compared with SAL-treated animals $\left(p<0.05 ; t_{(14)}=2.95\right.$, Fig. 2a) and a greater percentage decrease in social cage sniff time from baseline compared with SAL rats $\left(p<0.01 ; t_{(14)}=2.688\right.$, Fig. 2b). No difference was found in the total number of crossings made by rats of both groups between all the chambers at baseline (i.e., preinjection) $\left(p=0.11 ; t_{(14)}=1.718\right)$ or postinjection $(p=0.12$; $\left.t_{(14)}=1.629\right)$ (Fig. 2c).

Diazepam reverses increased anxiety-like behavior following acute withdrawal from a single dose of AMPH

Given the observed link between high anxiety levels and low social motivation in AMPH-withdrawn rats, we tested the ability of diazepam, an anxiolytic drug, to normalize anxiety-like behavior in the EPM in a separate cohort of animals (SAL-VEH: $n=8$; AMPHVEH: $n=7$; SAL-DZ: $n=6$; AMPH-DZ: $n=6$ ). SAL and AMPHwithdrawn rats treated with diazepam made fewer entries into the open arm [two-way ANOVA; main effect of diazepam $\left(F_{1,23}=6.82\right.$, $p<0.5$ ) (Fig. 3a)]. For time spent in open arms, there was an interaction between condition (i.e., SAL vs AMPH-withdrawn) and drug treatment (vehicle or diazepam) [two-way ANOVA $\left(F_{1,23}=\right.$ $7.07, p<0.5)$; and post hoc analysis revealed that AMPHwithdrawn rats treated with diazepam spent more time in the 
a

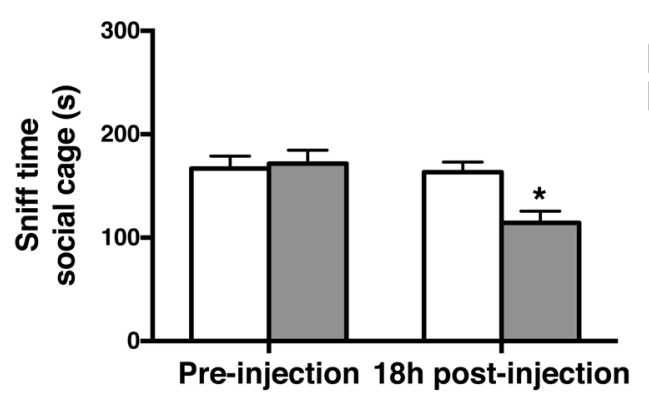

b Change from baseline

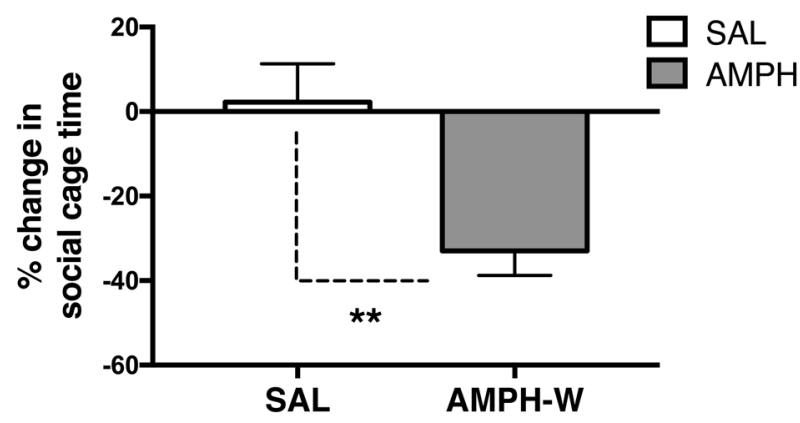

C

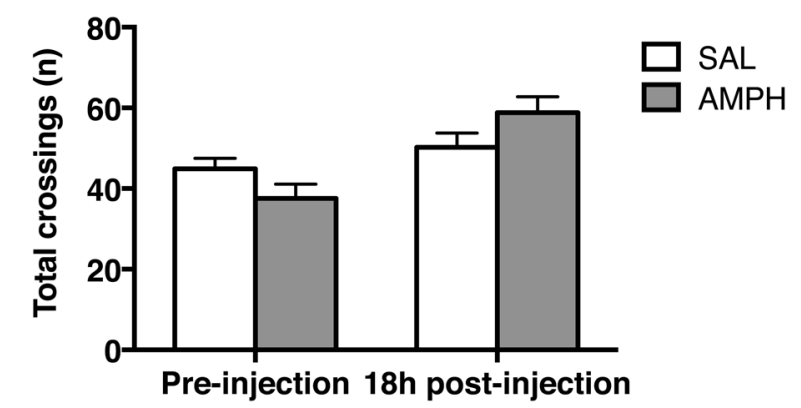

Fig. 2 Withdrawal from acute AMPH reduces social motivation. Animals were assigned to SAL $(n=9)$ or AMPH $(n=7)$ injection and tested for baseline social behavior prior to drug injection and retested $18 \mathrm{~h}$ postinjection to model AMPH-W. AMPH-withdrawn rats exhibited reduced social motivation, as indexed by a reduced sniff time of the cage containing the social stimulus animal $(p<0.05)$, and b a percentage decrease from baseline in sniff time of the social cage $(p<0.01)$. c No difference was found in the total number of crossings made by rats of both groups at preinjection $(p=0.11)$ or postinjection $(p=0.12) .{ }^{*} p<0.05,{ }^{* *} p<0.01$. Error bars indicate mean + SEM

open arms compared with vehicle-treated $\mathrm{AMPH}$-withdrawn rats (Tukey's multiple comparison post hoc test, $p<0.05$ ) (Fig. 3b). A diazepam effect was observed on the total number of entries in both SAL controls and AMPH-withdrawn rats, which exhibited a reduced total number of entries [2-way ANOVA; main effect of DZ: $\left.\left(F_{1,23}=18.01, p<0.001\right)\right]$ (Fig. 3c).

Diazepam normalizes social motivation and reverses DA neuron hypoactivity following acute withdrawal from a single dose of AMPH

The effects of diazepam on social behavior and VTA DA neuron activity were tested in a separate cohort of animals. At baseline (i.e., pretreatment), no difference in social sniff time was found

\section{EPM}
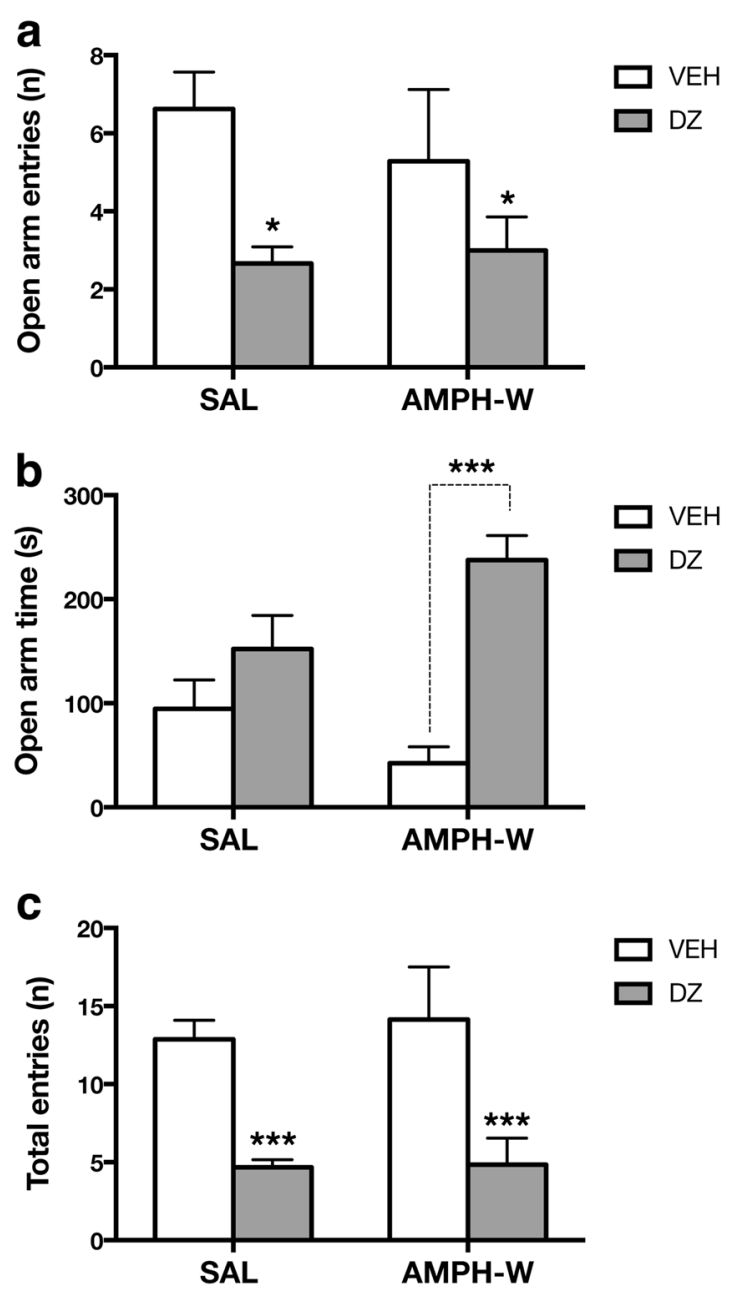

Fig. 3 Diazepam reverses increased anxiety-like behavior following acute AMPH withdrawal. a SAL and AMPH-W rats treated with diazepam (DZ) ( $n=6$ per group) made fewer entries into the open arm compared with vehicle-treated animals $(n=7-8$ per group; $p<$ 0.05). b AMPH-withdrawn rats treated with DZ spent more time in the open arms compared with vehicle-treated AMPH-withdrawn rats $(p<0.001)$. c DZ treatment reduced total number of entries in SAL and AMPH-W rats $(p<0.001) .{ }^{*} p<0.05,{ }^{* * *} p<0.001$. Error bars indicate mean + SEM

across treatment groups, although a treatment effect following AMPH-W was found [one-way ANOVA, pretreatment: $\left(F_{3,26}=0.03\right.$; $p=0.99)$, posttreatment: $\left(F_{3,26}=3.24, \quad p<0.05\right)$ Fig. $\left.4 a\right]$. Amphetamine-withdrawn rats treated with diazepam prior to social behavior testing exhibited increased social sniff time compared with vehicle-treated AMPH-withdrawn rats (Tukey's multiple comparison post hoc test, $p<0.05$ ). Moreover, there was an interaction between $\mathrm{AMPH}-\mathrm{W}$ and diazepam in the percentage change in social sniff time from baseline [two-way ANOVA: $\left(F_{1,26}=10.99 ; p<0.01\right)$, Fig. $\left.4 \mathrm{~b}\right]$. AMPH-withdrawn rats treated with diazepam did not exhibit the percentage decrease in social sniff time observed in AMPH-withdrawn rats treated with vehicle but instead exhibited an increase (Tukey's multiple comparison post hoc test, $p<0.05$ ). In terms of latency to contact social cage (see Supplementary Figure S2), we found no between-group differences at pretreatment $(p=0.36)$ or posttreatment $(p=0.55)$. The total number of chamber crossings was similar across treatment groups at baseline (i.e., pretreatment), although a diazepam effect was observed on the total number of crossings in both SAL and AMPH-withdrawn rats, which 
a Social Motivation

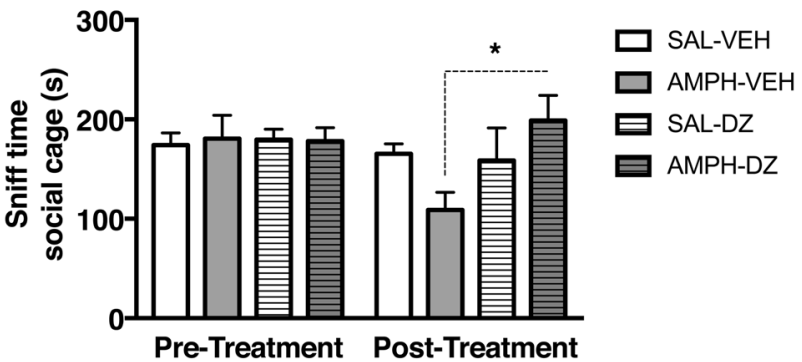

b Change from baseline

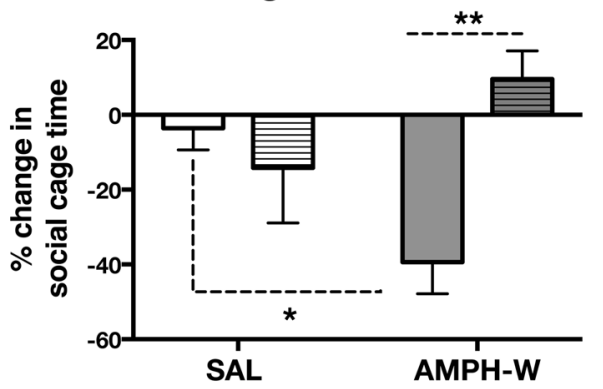

C

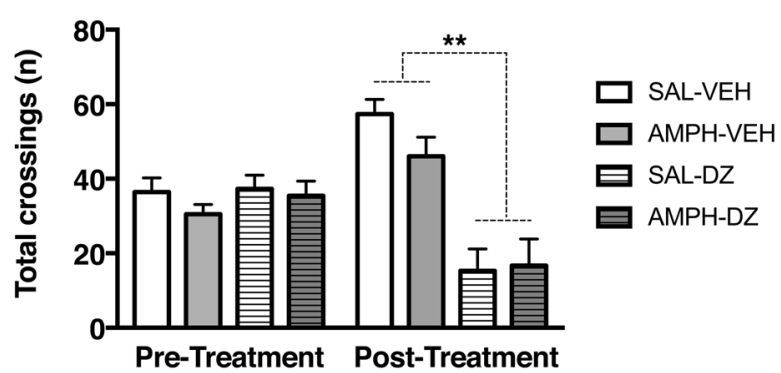

Fig. 4 Diazepam normalizes social motivation following withdrawal from acute AMPH. a No difference in social sniff time was found across treatment groups at baseline (i.e., pretreatment) $(p=0.99)$. Posttreatment, AMPH-withdrawn rats treated with DZ exhibited increased social cage sniff time compared with vehicle-treated AMPH-withdrawn rats $(p<0.05, n=6-9$ per group). b AMPHwithdrawn rats treated with vehicle exhibited a greater reduction in the percentage change of social motivation, as indexed by a greater percentage decrease in social sniff time, compared with non-withdrawn (i.e., SAL) vehicle-treated rats $(p<0.05)$. AMPHwithdrawn rats treated with $D Z$ exhibited a percentage increase in social sniff time $(p<0.01)$. c No difference was found in the total number of crossings made by rats of both groups at baseline $(p=$ 0.61 ), although a posttreatment effect of DZ was found in both SAL and AMPH-withdrawn rats compared with their vehicle-treated counterparts $(p<0.01)$. ${ }^{*} p<0.05,{ }^{* *} p<0.01$. Error bars indicate mean + SEM

exhibited a reduced total number of crossings [one-way ANOVA; pretreatment: $\left(F_{3,26}=0.61, p=0.61\right)$, posttreatment: $\left(F_{3,26}=13.92\right.$, $p<0.01)$ Fig. $4 c]$.

Extracellular recordings of VTA DA neurons were conducted in SAL controls and AMPH-withdrawn rats tested for social behavior after vehicle or diazepam treatment ( $n=7$ per group; SAL-VEH: 52 neurons, AMPH-VEH: 32 neurons, SAL-DZ: 49 neurons, AMPH-DZ: 41 neurons). An interaction between $\mathrm{AMPH}-\mathrm{W}$ and diazepam treatment $\left(F_{1,24}=4.905, p<0.05\right)$ was found for VTA population activity [two-way ANOVA: $\left(F_{1,24}=4.905, p<0.05\right)$; Fig. 5a]. Amphetamine-withdrawn rats treated with vehicle exhibited a reduction in the number of spontaneously firing VTA DA neurons
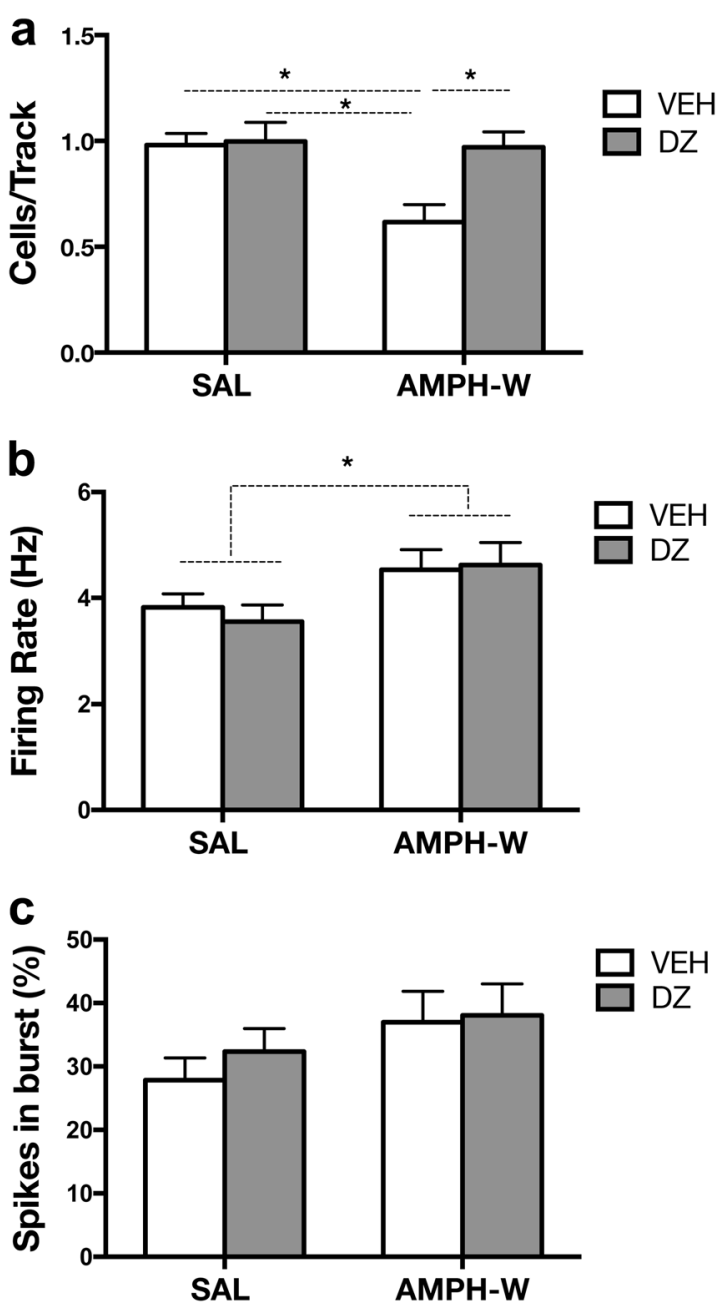

Fig. 5 Diazepam reverses the attenuation of VTA DA neuron activity induced by withdrawal from acute AMPH. Extracellular recordings of VTA DA neurons were conducted in SAL and AMPH-withdrawn rats following social behavior testing post-vehicle or DZ treatment $(n=7$ per group). a In contrast to AMPH-withdrawn rats receiving vehicle, AMPH-withdrawn rats treated with DZ did not exhibit an attenuation of DA neuron activity and instead showed population activity similar to SAL animals treated with vehicle or DZ $(p<0.05)$. b An increase in firing rate was observed in the AMPH-W groups regardless of vehicle or DZ treatment $(p<0.01)$. c No effect of AMPH-W $(p=0.08)$ or DZ $(p=0.51)$ was found in the percentage of spikes in burst. ${ }^{*} p<0.05,{ }^{* *} p<0.01$. Error bars indicate mean + SEM

(i.e., cells/track, CPT) compared with the control group (AMPHVEH: $0.59 \pm 0.17$ CPT vs SAL-VEH: $0.96 \pm 0.15$ CPT; $p<0.01$, Tukey's multiple comparison post hoc test). However, AMPH-withdrawn rats treated with diazepam did not exhibit an attenuation of DA neuron activity (AMPH-DZ: $0.95 \pm 0.22$ CPT vs AMPH-VEH: $0.59 \pm$ 0.17 CPT; $p<0.05$, Tukey's multiple comparison post hoc test) and instead showed population activity similar to control animals treated with vehicle or diazepam (SAL-VEH: $0.96 \pm 0.15 \mathrm{CPT}$, SALDZ: $0.92 \pm 0.23$ CPT). An increase in firing rate was observed in AMPH-withdrawn groups compared with control groups regardless of vehicle or diazepam treatment [two-way ANOVA: $\left(F_{1,170}=\right.$ $6.726, p<0.01)$; AMPH-VEH: $4.5 \pm 2.14 \mathrm{~Hz}$, AMPH-DZ: $4.6 \pm 2.73 \mathrm{~Hz}$; SAL-VEH: $3.82 \pm 1.85 \mathrm{~Hz}$, SAL-DZ: $3.5 \pm 2.19 \mathrm{~Hz}$; Fig. 5b). No effect of AMPH-W $\left(F_{1,170}=3.08, p=0.08\right)$ or diazepam $\left(F_{1,170}=0.4395, p=\right.$ 0.51 ) was found in the percentage of spikes in burst (SAL-VEH: $27.85 \pm 25.37 \%$; SAL-DZ: $32.35 \pm 25.35 \% \quad$ AMPH-VEH: $36.97 \pm$ 27.71\%; AMPH-DZ: $38.07 \pm 31.63 \%$; Fig. $5 \mathrm{C}$ ). 


\section{DISCUSSION}

The initial acute effect of a drug (i.e., positive hedonic response), including psychostimulants, is opposed or counteracted by longer-duration homeostatic changes in brain systems recruited by drug administration, thereby resulting in a longer-duration negative affective state upon withdrawal that is thought to contribute to the relapse and maintenance of addiction $[4,5,46]$. In the context of AMPH, this negative state appears even after initial acute AMPH administration and is accompanied by changes in both affective behavior and DA system activity [13].

Here we show that withdrawal from acute AMPH results in an anxiety-like state involving increases in anxiety-like behavior (i.e., less open arm entries, less time spent in open arms) and decreases in social motivation (i.e., reduced social cage sniff time). Increases in anxiety-like behavior are also observed in rats during short-term (24-48 h) withdrawal from chronic cocaine $[21,47]$ and AMPH [10]. In humans, acute withdrawal from psychostimulants is also accompanied by increased anxiety symptoms [17-19]. Collectively, these data suggest that both acute and repeated psychostimulant administration result in an increased anxiety-like state during the early withdrawal period. Our finding of social motivation impairment in AMPH-withdrawn rats, as indexed by lower levels of social sniff (i.e., cage) time, is consistent with prior reports, indicating suppression of rat social behaviors following acute AMPH administration [48, 49] and decreased motivation for natural reinforcers in animals undergoing $A M P H-W[50,51]$. Since both high anxiety and low social motivation were present in $\mathrm{AMPH}$-withdrawn rats, changes in social approach/social cage time likely reflect the anxiety state of the rat, suggesting that reducing the anxiety induced by $\mathrm{AMPH}-\mathrm{W}$ may correct the increased anxiety-like behavior and the social behavior deficit observed.

Diazepam, a drug known for its robust anti-anxiety effects, reversed the affective dysregulation induced by acute AMPH-W. Acute diazepam administration increased the time spent in the open arms in the EPM only in AMPH-withdrawn rats. Diazepam reduced the number of open arm entries and the total number of arm entries in AMPH-withdrawn and non-withdrawn (i.e., control) rats, suggesting that the drug exerted a sedative/hypolocomotor effect. This is consistent with a prior study indicating reduced locomotor activity in the EPM at higher doses in adult male Sprague Dawley rats [52]. Indeed, diazepam effects on locomotion were present in both AMPH-withdrawn and non-withdrawn rats, although a difference in open arm time was only found in diazepam treated rats undergoing $\mathrm{AMPH}-\mathrm{W}$, suggesting that effects of diazepam on locomotor activity did not drive the anxiolytic behavioral effects exerted by diazepam in this study. This is also consistent with a reduction in the total number of crossings in the social approach test in both diazepam-treated groups, in which the increase in social cage time was restricted to AMPH-withdrawn animals treated with diazepam. Thus, although diazepam reduced general locomotor activity in $\mathrm{AMPH}$-withdrawn and non-withdrawn rats in both behavioral tests, the anxiolytic effects were specific to the AMPH-withdrawn group. Importantly, diazepam increased social cage time in AMPH-withdrawn rats and prevented the percentage decrease from baseline in social cage time observed in $\mathrm{AMPH}$-withdrawn rats treated with vehicle while having no effect on SAL controls in these measures, suggesting a selective effect on social dysregulation induced by AMPH-W. Moreover, diazepam also resulted in an increase from baseline in social cage time only in AMPH-withdrawn animals. Taken together, these data suggest that diazepam not only neutralized the decrease in social motivation following withdrawal from acute AMPH but also enhanced social motivation. This agrees with several preclinical studies that have highlighted the ability of diazepam to positively modulate anxiety-like behavior and social impairments in rodent models of schizophrenia and autism, respectively $[36,53]$. For example, diazepam: (i) increases time spent in the social zone in $\mathrm{N}$-methyl-D-aspartate receptor-deficient mice, which normally show deficits in affiliative behaviors and reduced sociability [53]; (ii) increases time spent in the proximal social zone of the social-approach avoidance test in a high anxiety strain (F-344) of rats [54]; (iii) abolishes social avoidance induced by prior footshock exposure [37]; and (iv) facilitates social dominance in high anxious rats, which frequently show reduced social competitiveness and a subordinate status [55]. Altogether, these data indicate a link between anxiety and alterations within the social domain and suggest that reductions in social motivation can serve as a measure of anxiety in a social context, which is consistent with diazepam's anxiolytic effect on social behavior following AMPH-W, which was specific to the high anxiety group (i.e., AMPH-withdrawn rats).

Consistent with a prior study [13], we observed an attenuation of VTA population activity (i.e., DA cells/track) during withdrawal from acute AMPH. Thus motivational changes in behavior (i.e., anxiety, social dysfunction) during AMPH-W likely reflect opponent process-like changes (i.e., downregulation) in the reward circuitry initially activated by acute drug exposure. Reductions in VTA population activity have also been observed during shortterm withdrawal $(24 \mathrm{~h})$ from an escalating-dose, binge-like regimen of cocaine [56] and psychostimulant withdrawal results in marked reductions of mesolimbic DA release in rats [57]. Drug abstinence in psychostimulant users is associated with dopaminergic alterations including changes in DA release, transporter, and receptor availability [8]. Thus drug withdrawal-induced decreases in reward-related dopaminergic neurotransmission may reflect a within-system neuroadaptation and contribute significantly to the negative motivational state associated with drug abstinence, thereby making the mesolimbic DA system a possible target for modulating the negative affective state induced by drug withdrawal.

Diazepam reversed VTA DA hypofunction induced by AMPH-W and had a specific effect on VTA population activity following acute AMPH-W as it normalized the attenuation in the number of active DA cells, while having no effect on SAL controls. Amphetamine-withdrawn rats treated with diazepam did not exhibit the reduction in VTA population activity observed in vehicle-treated $A M P H$-withdrawn rats and instead had numbers of spontaneously active DA neurons that were comparable to the vehicle and diazepam-treated control (i.e., nonwithdrawn) groups. Diazepam has also been shown to attenuate neural alterations, as measured by Fos immunoreactivity, in response to chronic social defeat stress across multiple brain regions: prefrontal cortices, limbic structures, and midbrain structures including the VTA [58], suggesting that diazepam can reverse stress-induced patterns of neural activation. Notably, when placed in the context of our past work, the DA downregulation induced by AMPH-W is comparable to that observed in rats following exposure to other stressors (CMS, learned helplessness) [14, 16]. The decrease in VTA population activity in helpless rats is reversed by systemic ketamine administration, which normalizes synaptic activity within the ventral hippocampal-nucleus accumbens pathway to restore DA population activity [16]. Similarly, the reduction in VTA population activity induced by withdrawal from acute AMPH is also reversed by ketamine [13], implicating restored activity within this pathway as a potential mechanism. Since ketamine administration did not reverse increased immobility induced by withdrawal from acute AMPH, this suggested that VTA DA neuron activity and FST immobility are correlated but not interdependent and raised the possibility that different processes may be present during acute AMPH-W. Importantly, DA downregulation induced by withdrawal from acute AMPH is reversed by inactivation of the basolateral amygdala (BLA), and BLA hyperactivity is observed in rodent models of depression and schizophrenia involving increased anxiety $[14,36]$. Moreover, diazepam reduces anxietylike behavior and normalizes amygdala hyperactivity in the 
methylazoxymethanol acetate (MAM) model of schizophrenia [36]. Taken together, these data suggest that diazepam may normalize the anxiety-like state and social deficit in AMPHwithdrawn rats via inhibitory actions in the VTA, as diazepam mimicked the previously reported effects of BLA inactivation on VTA population activity [13]. A recent study identified the VTA as a key site of action for diazepam's enhancing effects on social competitiveness and dominance in high anxiety rodents [55]. Thus it is possible that diazepam reduces increased anxiety-like behavior and enhances motivated social behavior in AMPHwithdrawn rats through actions on the VTA, although this is a hypothesis that remains to be tested.

Finally, given that diazepam prevents a subset of the negative affective state symptoms resulting from acute withdrawal from a single dose of $\mathrm{AMPH}$, future studies could examine whether these findings extend to repeated (i.e., chronic) use and whether they have implications for future drug-seeking and/or self-stimulation behavior. Because transition from drug use to addiction is thought to result, at least in part, from the need to alleviate dysphoric states that emerge during drug withdrawal [4], if diazepam is effective at reducing the anxiety-like state following withdrawal from chronic use, then this may help break the cycle. However, this is a possibility that remains to be tested. In addition, stress exposure (i.e., social defeat) alters the mesolimbic DA system, including VTA DA activity, and increases the risk for addiction and escalated psychostimulant self-administration $[33,59,60]$. It would be interesting to see whether diazepam could circumvent or ameliorate the effects of stress on subsequent drug consumption.

In sum, the present data suggest that acute withdrawal from a single dose of AMPH is sufficient to induce an anxiety-like state characterized by enhanced anxiety-like behavior and social avoidance. These behavioral changes are accompanied by reduced activity within dopaminergic neurons of the VTA, suggesting that these neurons may play an important role in the affective dysregulation induced by $\mathrm{AMPH}-\mathrm{W}$. We tested whether increasing activity within this pathway (in conjunction with decreasing anxiety) would reverse the adverse behavioral effects precipitated by AMPH-W and found that the negative affective state resulting from $A M P H-W$ can be reversed by diazepam, which circumvents the decrease in tonic DA neuron firing that we propose underlies the negative withdrawal state. These data suggest that VTA DA neurons may be an appropriate target for modulating the negative affective state-induced withdrawal from acute AMPH.

\section{ACKNOWLEDGEMENTS}

We thank Niki MacMurdo and Christy Smolak for technical assistance with histology and Samuel B. Cartwright for assistance with behavioral scoring. Research reported in this publication was supported by the National Institute of Mental Health (NIMH) of the National Institute of Health (NIH) under award numbers T32-MH016804 and F32MH110128 to MR-C and R01-MH101180 to AAG.

\section{ADDITIONAL INFORMATION}

Supplementary Information accompanies this paper at (https://doi.org/10.1038/ s41386-018-0123-8).

Competing interests: AAG received consultant fees from Lundbeck, Pfizer, Otsuka, Asubio, Autofony, and Janssen and is on the advisory board for Alkermes, Newron, and Takeda. The other authors declare no competing interests.

Publisher's note: Springer Nature remains neutral with regard to jurisdictional claims in published maps and institutional affiliations.

\section{REFERENCES}

1. Koob GF. Neurobiology of addiction. Toward the development of new therapies. Ann NY Acad Sci. 2000;909:170-85.
2. Adinoff B. Neurobiologic processes in drug reward and addiction. Harv Rev Psychiatry. 2004;12:305-20.

3. Kalivas PW. Neurotransmitter regulation of dopamine neurons in the ventral tegmental area. Brain Res Brain Res Rev. 1993;18:75-113.

4. Koob GF, Le Moal M. Review. Neurobiological mechanisms for opponent motivational processes in addiction. Philos Trans R Soc Lond B Biol Sci. 2008;363: 3113-23.

5. Solomon RL, Corbit JD. An opponent-process theory of motivation. I. Temporal dynamics of affect. Psychol Rev. 1974;81:119-45.

6. Che Y, Cui YH, Tan H, Andreazza AC, Young LT, Wang JF. Abstinence from repeated amphetamine treatment induces depressive-like behaviors and oxidative damage in rat brain. Psychopharmacology (Berl). 2013;227:605-14.

7. Cryan JF, Hoyer D, Markou A. Withdrawal from chronic amphetamine induces depressive-like behavioral effects in rodents. Biol Psychiatry. 2003;54:49-58.

8. Ashok AH, Mizuno Y, Volkow ND, Howes OD. Association of stimulant use with dopaminergic alterations in users of cocaine, amphetamine, or methamphetamine: a systematic review and meta-analysis. JAMA Psychiatry. 2017;74:511-9.

9. Leventhal AM, Kahler CW, Ray LA, Stone K, Young D, Chelminski I, et al. Anhedonia and amotivation in psychiatric outpatients with fully remitted stimulant use disorder. Am J Addict. 2008;17:218-23.

10. Vuong SM, Oliver HA, Scholl JL, Oliver KM, Forster GL. Increased anxiety-like behavior of rats during amphetamine withdrawal is reversed by CRF2 receptor antagonism. Behav Brain Res. 2010;208:278-81.

11. Deckers L. Addictions and addictive behaiviors. motivation: biological, psychological and environmental. 4th ed. New York, NY: Routledge; 2016.

12. Koob GF, Le Moal M. Drug addiction, dysregulation of reward, and allostasis. Neuropsychopharmacology. 2001;24:97-129.

13. Belujon P, Jakobowski NL, Dollish HK, Grace AA. Withdrawal from acute amphetamine induces an amygdala-driven attenuation of dopamine neuron activity: reversal by ketamine. Neuropsychopharmacology. 2016;41:619-27.

14. Chang $\mathrm{CH}$, Grace AA. Amygdala-ventral pallidum pathway decreases dopamine activity after chronic mild stress in rats. Biol Psychiatry. 2014;76:223-30.

15. Rincon-Cortes M, Grace AA. Sex-dependent effects of stress on immobility behavior and VTA dopamine neuron activity: modulation by ketamine. Int J Neuropsychopharmacol. 2017;20(10):823-832.

16. Belujon P, Grace AA. Restoring mood balance in depression: ketamine reverses deficit in dopamine-dependent synaptic plasticity. Biol Psychiatry. 2014;76: 927-36.

17. Koob GF, Volkow ND. Neurocircuitry of addiction. Neuropsychopharmacology. 2010;35:217-38.

18. Lago JA, Kosten TR. Stimulant withdrawal. Addiction. 1994;89:1477-81.

19. Su H, Zhang J, Ren W, Xie Y, Tao J, Zhang X, et al. Anxiety level and correlates in methamphetamine-dependent patients during acute withdrawal. Medicine (Baltimore). 2017;96:e6434

20. Zorick T, Nestor L, Miotto K, Sugar C, Hellemann G, Scanlon G, et al. Withdrawal symptoms in abstinent methamphetamine-dependent subjects. Addiction. 2010;105:1809-18.

21. Sarnyai Z, Biro E, Gardi J, Vecsernyes M, Julesz J, Telegdy G. Brain corticotropinreleasing factor mediates 'anxiety-like' behavior induced by cocaine withdrawal in rats. Brain Res. 1995;675:89-97.

22. Allsop SA, Vander Weele CM, Wichmann R, Tye KM. Optogenetic insights on the relationship between anxiety-related behaviors and social deficits. Front Behav Neurosci. 2014;8:241.

23. Toth I, Neumann ID. Animal models of social avoidance and social fear. Cell Tissue Res. 2013;354:107-18.

24. Kroenke K, Spitzer RL, Williams JB, Monahan PO, Lowe B. Anxiety disorders in primary care: prevalence, impairment, comorbidity, and detection. Ann Intern Med. 2007;146:317-25.

25. Metzler DH, Mahoney D, Freedy JR. Anxiety disorders in primary care. Prim Care. 2016;43:245-61.

26. Calcaterra NE, Barrow JC. Classics in chemical neuroscience: diazepam (valium). ACS Chem Neurosci. 2014;5:253-60.

27. Jefferson JW. Benzodiazepines and anticonvulsants for social phobia (social anxiety disorder). J Clin Psychiatry. 2001;62:50-3.

28. Reinhold JA, Rickels K. Pharmacological treatment for generalized anxiety disorder in adults: an update. Expert Opin Pharmacother. 2015;16:1669-81.

29. Brady KT, Verduin ML, Tolliver BK. Treatment of patients comorbid for addiction and other psychiatric disorders. Curr Psychiatry Rep. 2007;9:374-80.

30. Kitanaka J, Kitanaka N, Takemura M. Neurochemical consequences of dysphoric state during amphetamine withdrawal in animal models: a review. Neurochem Res. 2008;33:204-19.

31. Gunaydin LA, Deisseroth K. Dopaminergic dynamics contributing to social behavior. Cold Spring Harb Symp Quant Biol. 2014;79:221-7. 
32. Hung LW, Neuner S, Polepalli JS, Beier KT, Wright M, Walsh JJ, et al. Gating of social reward by oxytocin in the ventral tegmental area. Science. 2017;357: 1406-11.

33. Yap JJ, Miczek KA. Stress and rodent models of drug addiction: role of VTA-accumbens-PFC-amygdala circuit. Drug Discov Today Dis Models. 2008;5:259-70.

34. Gil M, Nguyen NT, McDonald M, Albers HE. Social reward: interactions with social status, social communication, aggression, and associated neural activation in the ventral tegmental area. Eur J Neurosci. 2013;38:2308-18.

35. Kabitzke PA, Simpson EH, Kandel ER, Balsam PD. Social behavior in a genetic model of dopamine dysfunction at different neurodevelopmental time points. Genes Brain Behav. 2015;14:503-15.

36. Du Y, Grace AA. Amygdala hyperactivity in MAM model of schizophrenia is normalized by peripubertal diazepam administration. Neuropsychopharmacology. 2016;41:2455-62.

37. Leveleki C, Sziray N, Levay G, Barsvari B, Soproni K, Mikics E, et al. Pharmacological evaluation of the stress-induced social avoidance model of anxiety. Brain Res Bull. 2006;69:153-60.

38. Walf AA, Frye CA. The use of the elevated plus maze as an assay of anxietyrelated behavior in rodents. Nat Protoc. 2007;2:322-8.

39. Fairless AH, Shah RY, Guthrie AJ, Li H, Brodkin ES. Deconstructing sociability, an autism-relevant phenotype, in mouse models. Anat Rec (Hoboken). 2011;294: 1713-25.

40. Sandi C, Haller J. Stress and the social brain: behavioural effects and neurobiological mechanisms. Nat Rev Neurosci. 2015;16:290-304.

41. Hanks AN, Dlugolenski K, Hughes ZA, Seymour PA, Majchrzak MJ. Pharmacological disruption of mouse social approach behavior: relevance to negative symptoms of schizophrenia. Behav Brain Res. 2013;252:405-14.

42. Moy SS, Nonneman RJ, Shafer GO, Nikolova VD, Riddick NV, Agster KL, et al. Disruption of social approach by MK-801, amphetamine, and fluoxetine in adolescent C57BL/6J mice. Neurotoxicol Teratol. 2013;36:36-46.

43. Grace AA, Bunney BS. Intracellular and extracellular electrophysiology of nigral dopaminergic neurons--1. Identification and characterization. Neuroscience. 1983;10:301-15

44. Ungless MA, Grace AA. Are you or aren't you? Challenges associated with physiologically identifying dopamine neurons. Trends Neurosci. 2012;35:422-30.

45. Grace AA, Bunney BS. The control of firing pattern in nigral dopamine neurons: burst firing. J Neurosci. 1984;4:2877-90.

46. Barr AM, Markou A. Psychostimulant withdrawal as an inducing condition in animal models of depression. Neurosci Biobehav Rev. 2005;29:675-706.

47. Fung YK, Richard LA. Behavioural consequences of cocaine withdrawal in rats. J Pharm Pharmacol. 1994:46:150-2.
48. Li M, He W, Munro R. Differential effects of acute amphetamine and phencyclidine treatment and withdrawal from repeated amphetamine or phencyclidine treatment on social interaction and social memory in rats. PsyCh $\mathrm{J}$ 2012;1:56-68.

49. Steinpreis RE, Sokolowski JD, Papanikolaou A, Salamone JD. The effects of haloperidol and clozapine on PCP- and amphetamine-induced suppression of social behavior in the rat. Pharmacol Biochem Behav. 1994;47:579-85.

50. Barr AM, Fiorino DF, Phillips AG. Effects of withdrawal from an escalating dose schedule of d-amphetamine on sexual behavior in the male rat. Pharmacol Biochem Behav. 1999;64:597-604.

51. Barr AM, Phillips AG. Withdrawal following repeated exposure to D-amphetamine decreases responding for a sucrose solution as measured by a progressive ratio schedule of reinforcement. Psychopharmacology (Berl). 1999;141:99-106.

52. Mak P, Broussard C, Vacy K, Broadbear JH. Modulation of anxiety behavior in the elevated plus maze using peptidic oxytocin and vasopressin receptor ligands in the rat. J Psychopharmacol. 2012;26:532-42.

53. Mielnik CA, Horsfall W, Ramsey AJ. Diazepam improves aspects of social behaviour and neuron activation in NMDA receptor-deficient mice. Genes Brain Behav. 2014;13:592-602.

54. Nicolas LB, Prinssen EP. Social approach-avoidance behavior of a high-anxiety strain of rats: effects of benzodiazepine receptor ligands. Psychopharmacology (Berl). 2006;184:65-74.

55. van der Kooij MA, Hollis F, Lozano L, Zalachoras I, Abad S, Zanoletti O, et al. Diazepam actions in the VTA enhance social dominance and mitochondrial function in the nucleus accumbens by activation of dopamine D1 receptors. Mol Psychiatry. 2017;23(3):569-578.

56. Koeltzow TE, White FJ. Behavioral depression during cocaine withdrawal is associated with decreased spontaneous activity of ventral tegmental area dopamine neurons. Behav Neurosci. 2003;117:860-5.

57. Rossetti ZL, Hmaidan Y, Gessa GL. Marked inhibition of mesolimbic dopamine release: a common feature of ethanol, morphine, cocaine and amphetamine abstinence in rats. Eur J Pharmacol. 1992;221:227-34.

58. Lkhagvasuren B, Oka T, Nakamura Y, Hayashi H, Sudo N, Nakamura K. Distribution of Fos-immunoreactive cells in rat forebrain and midbrain following social defeat stress and diazepam treatment. Neuroscience. 2014;272:34-57.

59. Holly EN, Boyson CO, Montagud-Romero S, Stein DJ, Gobrogge KL, DeBold JF, et al. Episodic social stress-escalated cocaine self-administration: role of phasic and tonic corticotropin releasing factor in the anterior and posterior ventral tegmental area. J Neurosci. 2016;36:4093-105.

60. Boyson CO, Holly EN, Shimamoto A, Albrechet-Souza L, Weiner LA, DeBold JF, et al. Social stress and CRF-dopamine interactions in the VTA: role in long-term escalation of cocaine self-administration. J Neurosci. 2014;34:6659-67. 